Proceedings of the Workshop on Effective Tools and Methodologies for Teaching

Natural Language Processing and Computational Linguistics, Philadelphia,

July 2002, pp. 19-26. Association for Computational Linguistics.

\title{
A Web-based Instructional Platform for Constraint-Based Grammar Formalisms and Parsing
}

\author{
W. Detmar Meurers \\ Dept. of Linguistics \\ Ohio State University \\ dm@ling.osu.edu
}

\author{
Gerald Penn \\ Dept. of Computer Science \\ University of Toronto \\ gpenn@cs.toronto.edu
}

\author{
Frank Richter \\ Seminar für Sprachwissenschaft \\ Universität Tübingen \\ fr@sfs.uni-tuebingen.de
}

\begin{abstract}
We propose the creation of a web-based training framework comprising a set of topics that revolve around the use of feature structures as the core data structure in linguistic theory, its formal foundations, and its use in syntactic processing.
\end{abstract}

\section{Introduction}

Feature structures have been used prolifically at every level of linguistic theory, and they form the mathematical foundation of our most comprehensive and rigorous schools of syntactic theory, including Lexical-Functional Grammar and Head-driven Phrase Structure Grammar. This data structure is popular because it shares many properties with the first-order terms of classical logic, and in addition provides named access to substructures through paths of features. Often it also includes a type system reminiscent of the taxonomical classification systems that are widely used in knowledge representation, psychology and the natural sciences.

For teaching a subject like computational linguistics, which draws on a broad curriculum from many traditional disciplines to audiences with mixed backgrounds themselves, feature-structure-based theoretical and computational linguistics have three important properties. First, they are a mature discipline, in which a great deal of accomplishments have been made over the last 20 years, spanning from empirical and conceptual advances in linguistic theory to its mathematical and computational foundations, to grammar development and efficient processing.
Second, they are pervasive as an already existing representation standard for many levels of linguistic study. Third, they are transparent, reducing complex theories of grammar to a basic collection of mathematical concepts and algorithms for answering formal questions about those theories. One can address the distinction between descriptions of objects and the objects themselves, the difference between consistency and truth, and what it means for a syntactic theory to be not only elegant but correct in a precise and provable sense.

The purpose of this paper is to discuss how these three properties can be cast into an instructional setting to arrive at a framework for teaching computational linguistics that highlights the integrated nature and precision with which work in this very heterogeneous discipline can be presented. In principle, the framework we are proposing is open-ended, in the sense that additional modules should be added by students and other researchers, subject to the design principles given in Section 3. We are currently designing three of the core modules for this framework: formal foundations, constraint-based grammar implementation, and parsing.

\section{Problems of seminar-style courses}

The contents of our core modules are based on a series of previous seminar-style courses, in particular on constraint-based grammar implementation, which also started integrating interactive components and web-based materials into traditional faceto-face teaching. These are described in detail in Section 5. The traditional seminar-style teaching method underlying the courses mentioned therein 
has a number of inherent problems, however. These problems become particularly pressing when topics as diverse as linguistic theory, grammar implementation, parsing, mathematical foundations of linguistic theory and feature logics are combined in a single course that is addressed to a mixed audience with varying backgrounds in computer science, knowledge representation, artificial intelligence and linguistics, in any combination of these subjects.

First, the seminar-style teaching format as used in those grammar implementation courses presupposes a fairly coherent audience of linguists with a shared background of linguistic knowledge. Second, since computers are only used as a medium to implement grammars and since the implementation platform is not optimized for web-based training, it is necessary that there be a relatively low number of students per teacher. Third, the theoretical material is in the form of overheads and research papers, which are in electronic form but not easily accessible without the accompanying lecture as part of a seminarstyle course. Fourth, the background lectures of the courses lack the support of the kind of graphical, interactive visualization that teaching software can in principle offer. Finally, the courses follow a single path through the materials as determined by the teacher, which the student cannot change according to their specific interests and their prior knowledge.

We believe that these shortcomings can be overcome by shifting from a seminar-style to a webbased training format in a way that preserves the positive aspects of successful hands-on courses. On the other hand, to successfully shift from seminarstyle to web-based training we believe it is essential to do this based on a scientific understanding of the nature and possibilities of web-based learning. In the next section we therefore embed our work in the context of education and collaborate learning technology research.

\section{Education and collaborative learning technology research}

Our perspective on web-based training draws its inspiration primarily from work in building "learning communities" in education research (Lin et al., 1995; Nonaka, 1994), in which:

1. a precise context is established to introduce tacit knowledge and experience, in this case on subjects in computational linguistics and the traditional disciplines it draws from,

2. conflicting perspectives are shared, concepts are objectified and submitted to a process of justification and arbitration, and

3. the concepts are then integrated into the knowledge base as modules upon which further instructional material or grammar implementations can be constructed.

We thus intend to provide an environment that teaches students by actively encouraging them to participate in research that extends our collective knowledge in this area. In principle, there are no boundaries to the material that could be included in the evolving framework. We intend to make it available as an open-source standard for grammar development and instruction in the hope that this will encourage researchers and educators to contribute modules to it, and to use a feature-structure based approach for their own research and courses.

Scardamalia and Bereiter (1993) identify seven global characteristics that technologies must have to support this kind of participation:

Balance: a distinction between public and private and between individual and group knowledge processes. That includes free access to others' work, including implementations of concepts as algorithms or grammars, and opportunities to borrow ideas into their own work that would be prohibitively timeconsuming or otherwise advanced to formulate on their own. Such technologies must also encourage time for personal "reflection and refinement" and anonymous public or private contribution to the knowledge space. The present framework achieves this by providing an open-source setting combined with a web-based instructional tool for self-paced learning and individual design of both the contents and order of the curriculum.

Contribution and notification: to prevent ideas from being presented in an insulated structure that discourages questioning, debate, or revision. As discussed in Section 4.2, this is achieved by providing extensive linking and annotation of resources using web-compatible metalanguages for integrating mod- 
ules at the implementational, formal and instructional levels.

Source referencing: a means of preserving the boundaries of a contributor's idea and its credit as well as a history of prior accounts and antecedents to the idea. In the present framework, this is provided by means of a requirements analysis component that requires contributed modules to identify the contribution by new concepts or resources provided, existing concepts or resources imported for it to work, and an account of existing alternatives with a description of its distinction from them.

Storage and retrieval: which places contributions in a "communal context" of related contributions by others to encourage joint work between contributors working on problems with significant overlap. The present framework must organize the presentation of existing modules along several thematic dimensions to accomplish this.

Multiple points of entry: for students/contributors with different backgrounds and levels of experience. Material is made accessible in more basic or fundamental modules by projecting the formal content of the subject into a graphically based common-sense domain at which it can be grasped more intuitively (see Section 4.3). Accessibility in more advanced modules is provided by links specified in the requirements analysis component to more basic modules that the former rely upon.

Coherence-producing mechanisms: feedback to contributors and framework moderators of modules that are "fading" for lack of attention or further development. These can either be reinstated or reformulated, moved to a private space of more peripheral modules, or deleted outright. This is a way of encouraging activity that is productive, and restricting the chance of confusion or information overload. Such a coherence mechanism must exist within this framework.

Links to external resources: to situate the justification and discussion of contributions in a wide context. We make use of the web-based training platform ILIAS ${ }^{1}$ which is available as open source software and offers a high degree of flexibility in terms of the integration of internal and external resources.

\footnotetext{
${ }^{1}$ http://www.ilias.uni-koeln.de/ios/index-e.html
}

\section{Integration of the framework}

The goal of our current work is to transform previous, seminar-style courses and new input into teaching materials that are fit for web-based training in the general framework outlined in the previous section. This clearly involves much more than simply reformatting old teaching materials into web-compatible formats. Instead, it requires an analysis of the contents of the courses, the interleaving and hyperlinking of the textual materials, and the development of graphical, interactive solutions for presenting and interacting with the content of the material. Since the nature of the textual material as such is familiar (instructional notes, reference guides to major sections with indices, system documentation, annotated system source code, and annotated grammar source code), we use the limited space in this paper to highlight the integrated nature of the approach as well as the web-based training specific issues of hyperlinking and visualization.

\subsection{Integration of linguistic and computational aspects}

Our approach is distinguished by its integration of grammars, the parsers that use them and the online instructional materials. Compared to the LKB system $^{2}$, which as mentioned in Section 5.2 has also been used successfully in teaching grammar development, the greater range of formal expressive devices available to our parsing system, called TRALE, allows for more readable and compact grammars, which we believe to be of central importance in a teaching context. To illustrate this, we are currently porting the LinGO ${ }^{3}$ English Resource Grammar (ERG) from the LKB (on which the ERG was designed) to the TRALE system.

Given the scope of our web-based training framework as including an integrated module on parsing, it is also relevant that the TRALE system itself can be relatively compact and transparent at the sourcecode level since it exploits its close affinity to the underlying Prolog on which it is implemented. This contrasts with the perspective of Copestake et al. (2001), who concede that the LKB is unsuitable for teaching parsing.

\footnotetext{
${ }^{2} \mathrm{http} / / / \mathrm{www}$-csli.stanford.edu/ aac/lkb.html

${ }^{3}$ http://lingo.stanford.edu/csli/
} 


\subsection{The use of hyperlinks}

Several different varieties of links are distinguished within the course material, giving a first-class representation to the transfer of knowledge between the linguistic, computational and mathematical sources that inform this interdisciplinary area. We intend to distinguish the following kinds of links:

Conceptual/taxonomical: connecting instances of key concepts and terms used throughout the course material with their definitions and provenience;

Empirical context: connecting instances of design decisions, algorithms and formal definitions to encyclopedic discussions of their linguistic motivation and empirical significance;

Denotational: connecting instances of constructional terms and issues within linguistics as well as correctness conditions of algorithms to the mathematical definitions that formalize them within the foundations of constraint-based linguistics;

Operational: connecting mathematical definitions and instances of related linguistic discussions to computational instructional material describing the algorithms used to construct, refute or transform the formal objects representing them in a practical system;

Implementational: connecting discussions of algorithms to the actual annotated system source code in the TRALE system used to implement them, and mathematical definitions and discussions of linguistic constructions to the actual annotated grammar source code used to represent them in a typical implementation.

The idea behind this classification is that when more course material is added to the web-based training framework we are proposing, the new material will take into account these distinctions to obtain a conceptually coherent use of hyperlinks throughout the framework.

\subsection{Visualization}

Our three core modules make use of a number of graphical user interfaces: a tool for interleaved visualization and interaction with trees and attribute value matrices, one for the presentation of lexical rules and their interaction, an Emacs-based sourcelevel debugger, and a program for the graphical exploration of the formal foundations of typed feature logic. The first two are extensions of tools we already used for our previous courses, and the third is an extension of the ALE source-level debugger, so we here focus on the last, new development.

The main goal of the MorphMoulder (MoMo) is to project the formality of its subject, the formal foundations of constraint languages over typed feature structures, onto a graphical level at which it can be grasped more intuitively. ${ }^{4}$ The transparency of this level is essential for providing multiple points of entry (Section 3) to this fundamentally important module. The MoMo tool allows the user to explore the relationship between the two levels of the formal architecture: the descriptions and the elements described. To this end, the user works with a graphical interface on a whiteboard. Labeled directed graphs representing feature structures can be constructed on the whiteboard from their basic components, nodes and arcs. The nodes are depicted as colored balls, which are assigned types, and the arcs are depicted as arrows that may be labeled by feature names. Once a feature structure has been constructed, the user may examine its logical properties. The three main functions of the MoMo tool allow one to check (1) whether a feature structure complies with a given signature, (2) whether a wellformed feature structure satisfies a description or a set of descriptions, and (3) whether a well-formed feature structure is a model of a description or a set of descriptions. In the context of the course, the functions of MoMo thus lead the user from understanding the well-formedness of feature structures with respect to a signature to an understanding of feature structures in their role as a logical model of a theory. If a student has chosen course modules that include a focus on formal foundations of feature logics or feature logics based linguistic theory, the first introduction to the subject by MoMo can easily be followed up by a course module with rigorous mathematical definitions.

In constraint-based frameworks, the user declares the primitives of the empirical domain in terms of a type hierarchy with appropriate attributes and attribute values. Consider a signature that licenses lists of various birds, which may then be classified according to certain properties. First of all, the sig-

\footnotetext{
${ }^{4}$ MoMo is written by Ekaterina Ovchinnikova, U. Tübingen.
} 
nature needs to comprise a type hierarchy and feature appropriateness conditions for lists. Let type list be an immediate supertype of the types non-emptylist and empty-list in the type hierarchy (henceforth abbreviated as nelist and elist). Let the appropriateness conditions declare the attributes HEAD and TAIL appropriate for (objects of) type nelist, the values of TAIL at nelist be of type list, and the values of HEAD at type nelist be of type bird (for lists of birds). Finally no attributes are appropriate for the type elist. A typical choice for the interpretation of that kind of signature in constraint-based formalisms is the collection of totally well-typed and sort resolved feature structures. All nodes of totally welltyped and sort resolved feature structures are of a maximally specific type (types with no subtypes); and they have outgoing arcs for all and only those features that are appropriate to their type, with the feature values again obeying appropriateness. Our signature for lists thus declares an ontology of feature structures with nodes of type nelist or elist (but never of type list), where the former must bear the outgoing arcs HEAD and TAIL, and the latter have no outgoing arcs. They signal the end of the list. The HEAD values of non-empty lists must be in the denotation of the type bird.

Figure 1 illustrates how the MoMo tool can be used to study the relationship between signatures and the feature structures they license by letting the user construct feature structures and interactively explore whether particular feature structures are well-formed according to the signature. To the left of the whiteboard there are two clickable graphics consoles of possible nodes and arcs from which the user may choose to draw feature structures. The consoles offer nodes of all maximally specific types and arcs of all attributes that are declared in the signature. In the present example, parrot, woodpecker, and canary are the maximally specific subtypes of bird.

Each color of edge represents a different attribute, and each color of node represents a different type. The grayed outlines on edges and nodes indicate that all of the respective edges and nodes in this particular example are licensed by the signature that was provided. The HEAD arc originating at the node of type elist, however, violates the appropriateness conditions of the signature. The feature structure de-

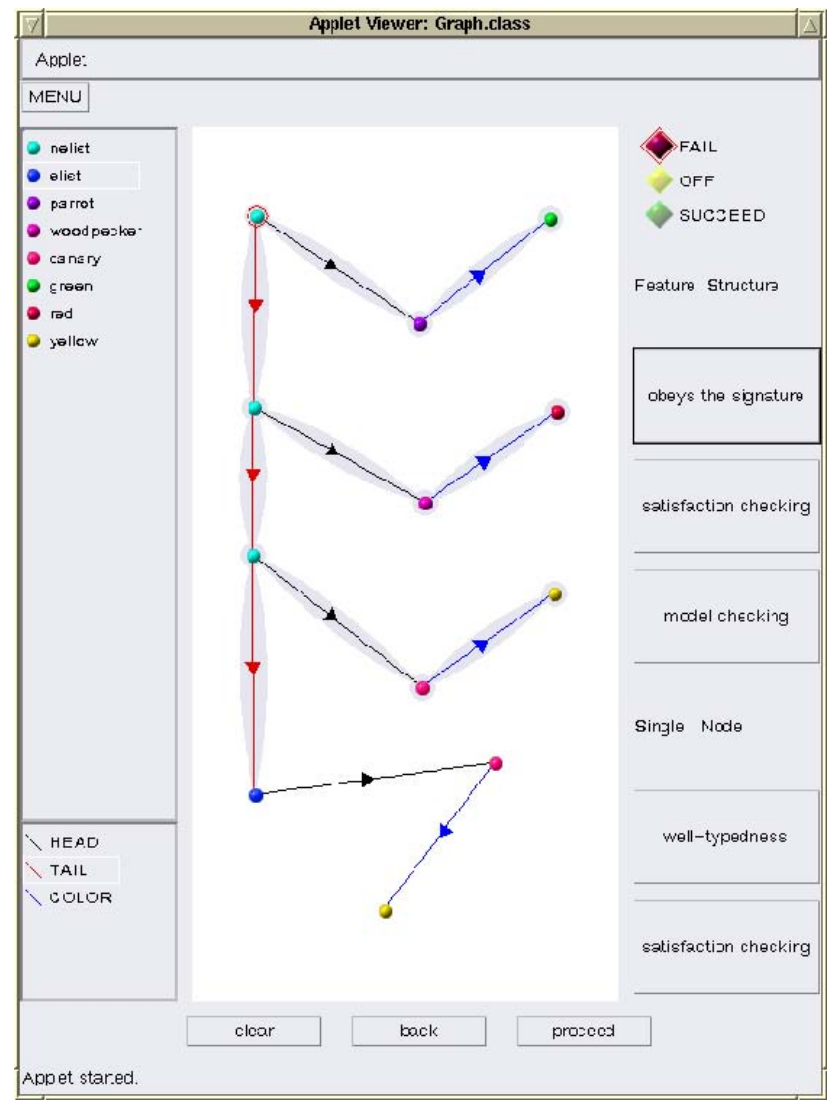

Figure 1: Graphically evaluating well-typedness of feature structures.

picted here, therefore, is not well-formed. The signature check thus fails on the given feature structure, as indicated by the red light in the upper function console to the right of the whiteboard.

Similarly, MoMo can graphically depict satisfiability and modellability of a single description or set of descriptions. To this end, the user may be asked to construct a description that a given feature structure satisfies or models; or she may be asked to construct feature structures that satisfy or model a given description (or set of descriptions). The system will give systematic feedback on the correct or incorrect usage of the syntax of the description language as well as on to which extent a feature structure satisfies or models descriptions, systematically guiding the user to correct solutions.

Figure 2 shows a successful satisfiability check of a well-formed feature structure. The feature structure is derived from the one in Figure 1 by removing the incorrect HEAD arc and its substructure 
from the elist node. The query, asked in a separate window, is whether the feature structure satisfies the constraint (nelist, head: (parrot, color:green), tail:nelist). Since this is the case, the green light on the function console to the right is signaling succeed. If we were to perform model checking of the same feature structure against the same constraint, checking would fail, and MoMo would indicate the nodes of the feature structure that do not satisfy the given constraint.

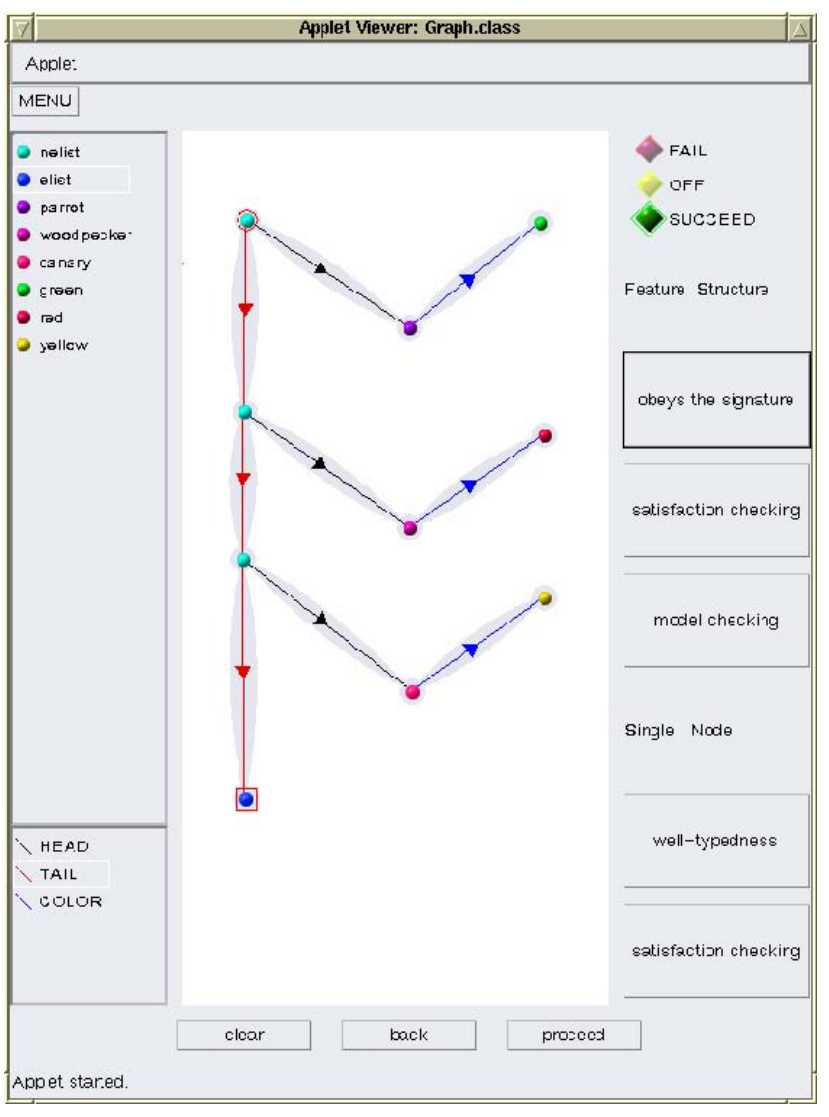

Figure 2: Graphically evaluating constraint satisfaction of feature structures.

MoMo's descriptions are a syntactic parallel to TRALE's descriptions, thus introducing the student not only to the syntax and semantics of constraint languages but also to the language that will be used for the implementation of grammars later in the course. The close relationship of description languages also facilitates a comparison of their modeltheoretic semantics and the truth conditions of grammars with the structure and semantics of algorithms that use descriptions for constraint resolution and in parsing. Finally, their common structure allows for a tight network of hyperlinks across the boundaries of different course modules and course topics, linking them to a common source of mathematical, implementational and linguistic indices, which explain the usage of common mathematical concepts across the different areas of application of typed feature structures.

\section{From seminar-style courses to web-based training}

Having discussed the ideas driving the web-based teaching platform and exemplified one of the tools, we now return to the courses which have informed our work on the three core modules currently being developed in terms of their content and the use of a web- and implementation environment they make.

\subsection{Grammar implementation in ALE}

ALE $^{5}$ (Carpenter and Penn, 1996) is a conservative extension of Prolog based on typed feature structures, with a built-in parser and semantic-headdriven generator. The demand for such a utility was so great when it was beta-released in 1992 that it immediately became the subject of early work in graphical front-end development for large constraint-based grammars: first with the Pleuk system (Calder, 1993), then as one of several systems supported by Gertjan van Noord's HDrug ${ }^{6}$, followed by an ALE-mode Emacs user interface (Laurens, 1995). It also provided the computational support for one of the very first web-based computational linguistics courses, Colin Matheson's widely used HPSG Development in ALE ${ }^{7}$. A follow-up course on computational morphology ${ }^{8}$, also by Colin Matheson, was based on ALE-RA ${ }^{9}$, a morphological extension of ALE by Tomaz Erjavec.

Our current web-based training module is supported by an extension of ALE, called TRALE, that uses a slightly different interpretation of typing found in many linguistic theories and an enhanced constraint language that supports constraints with complex antecedents (Penn, 2000).

\footnotetext{
${ }^{5}$ http://www.cs.toronto.edu/ $/$ gpenn/ale.html

${ }^{6} \mathrm{http} / /$ grid.let.rug.nl/ vannoord/hdrug/

${ }^{7}$ http://www.ltg.hcrc.ed.ac.uk/projects/ledtools/ale-hpsg/

${ }^{8} \mathrm{http}: / / w w w . l t g . e d . a c . u k / p r o j e c t s / l e d t o o l s / a l e-r a /$

${ }^{9}$ http://nl.ijs.si/et/Thesis/ALE-RA/
} 


\subsection{Constraint-based grammar implementation}

Over the past five years, we have held another course on Constraint-Based Grammar Implementation in a variety of settings, from summer schools to regular curriculum courses. ${ }^{10}$ It offers hands-on experience to linguists interested in the formalization of linguistic knowledge in a constraint-based grammar formalism. The course is taught in an interactive fashion in a computer laboratory and combines background lectures with practical exercises on how to specify grammars in ConTroll ${ }^{11}$ (Götz and Meurers, 1997), a processing system for constraint-based grammars intended to process with HPSG theories directly from the form in which they are constructed by linguists.

The background lectures of the Constraint-based grammar implementation courses introduce the relevant mathematical and computational knowledge and focus on the main ingredients of constraintbased grammars: highly structured lexical representations, constituent structures, and the encoding of well-formedness constraints on grammatical representations. In the lab, students work on exercises exploring the theoretical concepts covered in the lectures. In a later part of the course, they are given the opportunity to undertake individualized grammar projects for modeling theoretically and empirically significant syntactic constructions of their native language.

This course was the first hands-on computational syntax course at the European Summer School in Language, Logic, and Information (ESSLLI, 1997: Aix-en-Provence), and was also offered at the LSA Linguistic Institute (1999: University of Illinois, Urbana-Champaign) ${ }^{12}$ and the Computational Linguistics and Represented Knowledge (CLaRK) Summer School (1999: Eberhard-Karls Universität, Tübingen $)^{13}$. Generally regarded as a highly successful course and teaching method, every subsequent ESSLLI summer school has offered at least one similar course: Practical HPSG Grammar Engineering (1998: Ann Copestake, Dan Flickinger, and

\footnotetext{
${ }^{10}$ The courses were taught by E. Hinrichs and D. Meurers.

${ }^{11} \mathrm{http}: / / \mathrm{www} . \mathrm{sfs}$.uni-tuebingen.de/controll/

${ }^{12}$ http://ling.osu.edu/ dm/lehre/lsa99/

${ }^{13}$ http://ling.osu.edu/ dm/lehre/clark99/
}

Stephan Oepen $)^{14}$, Development of large scale LFG grammars: Linguistics, Engineering and Resources (1999: Miriam Butt, Annette Frank, and Jonas Kuhn $)^{15}$, Grammatical Resources: Logic, Structure, Control (1999: Michael Moortgat and Richard T. Oehrle $)^{16}$, An Introduction to Grammar Engineering using HPSG (2000: Ann Copestake, Rob Malouf $)^{17}$, Advanced Grammar Engineering using HPSG (2000: Dan Flickinger, Stephan Oepen) ${ }^{18}$, and An Introduction to Stochastic Attribute-Value Grammars (2001: Rob Malouf, Miles Osborne) ${ }^{19}$.

\subsection{Introduction to theory-driven $\mathbf{C L}$}

A further source of material for the core modules of our web-based training framework is the graduate level Introduction to Theory-driven Computational Linguistics at the Ohio State University. ${ }^{20}$ It covers the basic issues of the following topics: finite state automata and transducers, formal language theory, computability and complexity, recognizers/parsers for context free grammars, memoization, and parsing with complex categories.

The theoretical material is combined with practical exercises in Prolog implementing different aspects of parsers. At the end of the course, students complete a project consisting of building and testing a grammar fragment for a short English text of their choice. The traditional one-quarter course includes weekly exercises, extensive web-based course material for students, and a course workbook ${ }^{21}$ as a guide through the theoretical material.

\subsection{Model-theoretic introduction to Syntax}

Our approach to teaching the fundamentals of mathematical theories through graphical metaphors in the context of syntax derives from our experience with this method in teaching Syntax I (HPSG) at the Eberhard-Karls Universität Tübingen in 1998,

\footnotetext{
${ }^{14} \mathrm{http} / / / \mathrm{www} . c o l i . u n i-s b . d e / e s s l l i / S e i t e n / O e p e n . h t m l$

${ }^{15}$ http://www.let.uu.nl/esslli/Courses/butt.html

${ }^{16} \mathrm{http}: / /$ www.let.uu.nl/esslli/Courses/moortgat-oehrle.html

${ }^{17} \mathrm{http}: / /$ www.cs.bham.ac.uk/ esslli/notes/copestake.html

${ }^{18} \mathrm{http} / / /$ www.cs.bham.ac.uk/ esslli/notes/oepen.html

${ }^{19}$ http://odur.let.rug.nl/ malouf/esslli01/

${ }^{20}$ The course was taught by D. Meurers; see http://ling.osu. $\mathrm{edu} / \sim \mathrm{dm} / 2001 /$ winter/684.01/

${ }^{21}$ This workbook is based, with kind permission from the authors, on the module workbook for "Techniques in Natural Language Processing 1" by Chris Mellish, Pete Whitelock and Graeme Ritchie, 1994, Dept. of AI, University of Edinburgh.
} 
1999 and 2001.22 In these seminars, which did not presuppose any prior knowledge of model-theoretic methods in logic, the mathematical foundations of feature logic were introduced by intuitive means but with as much precision as possible without strict formalization. An introduction to a standardized version of the logical description language of HPSG was accompanied with problem sets that required the students to construct three-dimensional feature structure models (made of styrofoam and wires) of descriptions and sets of descriptions. The informal but very concrete understanding of the relationship between a theory cast in a constraint language and its feature structure models had a very positive result on students' ability to grasp and build working analyses of unseen constructions compared to the results of the more traditional method of teaching constraintbased syntax used in previous years. At the same time, the teaching method successfully used an appeal to prior world knowledge rather than unfamiliar mathematical notation in order to make the students familiar with the basic concepts of constraint satisfaction and truth in feature logics.

\section{Summary and Outlook}

The interdisciplinary nature of computational linguistics and the diverse backgrounds of the student audience makes it particularly attractive to teach a subject like constraint-based grammar formalisms and parsing using a web-based instructional platform which integrates formal and computational foundations, linguistic theory, and grammar implementation. We discussed several seminar-style courses which have informed our proposal in terms of content, highlighted the problems of the traditional face-to-face teaching, and described our environment of web-based teaching materials plus implementational support. We argued that a web-based training framework for the topic can be organized around feature structures as a central data structure in formal foundations, linguistics and implementation. We outlined the educational and collaborative learning background in which an informed proposal on web-based training must be embedded and used the newly developed tool MoMo as an illustration

\footnotetext{
${ }^{22}$ The courses were taught by F. Richter and M. Sailer; see http://www.sfs.uni-tuebingen.de/ /fr/teaching/
}

of how we envisage projecting the formal content of the subject into a graphically based common-sense domain in which it can be grasped more intuitively.

The three core modules on formal foundations, constraint-based grammar implementation, and parsing will be completed and made publicly available at the end of 2003. The joint project is funded by the German Federal Ministry for Research Technology (BMBF) as part of the consortium Media-intensive teaching modules in the computational linguistics curriculum (MiLCA). ${ }^{23}$

\section{References}

J. Calder. 1993. Graphical interaction with constraintbased grammars. In Proceedings of PACLING '93, pages 160-168, Vancouver, British Columbia.

B. Carpenter and G. Penn. 1996. Compiling typed attribute-value logic grammars. In H. Bunt and M. Tomita, editors, Recent Advances in Parsing Technologies, pages 145-168. Kluwer, Dordrecht.

A. Copestake, J. Carroll, D. Flickinger, R. Malouf, and S. Oepen. 2001. Using an open-source unificationbased system for CL/NLP teaching. In Proceedings of the EACL/ACL Workshop on Sharing Tools and Resources for Research and Education, pages 35-38.

T. Götz and W. D. Meurers. 1997. The ConTroll system as large grammar development platform. In Proceedings of the EACL/ACL Workshop on Computational Environments for Grammar Development and Linguistic Engineering, pages 38-45. http://ling.osu.edu/ $/ \mathrm{dm} /$ papers/envgram.html.

O. Laurens. 1995. An Emacs user interface for ALE. Technical Report CSS-IS TR 95-07, School of Computing Science, Simon Fraser University.

X. Lin, J.D. Bransford, and C.E. Hmelo. 1995. Instructional design and development of learning communities: an invitation to dialogue. Educational Technology, 35(5):53-63.

I. Nonaka. 1994. A dynamic theory of organizational knowledge creation. Organizational Science, 5(1).

G. Penn. 2000. Applying Constraint Handling Rules to HPSG. In Proceedings of the Workshop on RuleBased Constraint Reasoning and Programming, CL 2000.

M. Scardamalia and C. Bereiter. 1993. Technologies for knowledge-building discourse. Communications of the ACM, 36(5):37-41.

\footnotetext{
${ }^{23} \mathrm{http}: / /$ milca.sfs.uni-tuebingen.de/A4/HomePage/top.html
} 